\title{
Survey on Different Image Fusion Techniques
}

\author{
Miss. Suvarna A. Wakure ${ }^{1}$, Mr. S.R. Todmal ${ }^{2}$ \\ ${ }^{1}$ M.E.(E\&TC-Signal Processing), ${ }^{2}$ HOD(IT Department) University of Pune \\ ${ }^{1,2}$ JSPM's, ICOER, Pune, India
}

\begin{abstract}
In medical imaging and remote sensing, image fusion technique is a useful tool used to fuse high spatial resolution panchromatic images (PAN) with lower spatial resolution multispectral images $(M S)$ to create a high spatial resolution multispectral of image fusion while preserving the spectral information in the multispectral image (MS).Image fusion is the process that combines information from multiple images of the samescene. The result of image fusion is a new image that retains the most desirable informationand characteristics of each input image. Now-a-days, almost all areas of medical diagnosis are impacted by the digital image processing. When an image is processed for visual interpretation, the human eye is the judge of how well a particular method works. Clinical application demanding Radiotherapy plan, for instance, often benefits from the complementary information in images of different modalities. For medical diagnosis, Magnetic Resonance Image (MRI) is a medical imaging technique used in radiology to visualize internal structures of the body in detail. MRI provides better information on soft tissue with more distortion. Whereas, Computed Tomography (CT) provides the best information on denser tissue with less distortion. Wavelet transform fusion is more formally defined by considering the wavelet transforms of the two registered input images together with the fusion rule.Then, the inverse wavelet transform is computed, and the fused image is reconstructed. The wavelets used in image fusion can be classified into three categories Orthogonal, Bi-orthogonal and A 'trous' wavelet. Although these wavelets share some common properties, each wavelet has a unique image decompression and reconstruction characteristics that lead to different fusion results. Since medical images have several objects and curved shapes, it is expected that the curvelet transform would be better in their fusion. In this paper the fusion results are compared visually and statistically. The simulation results show the superiority of the curvelet transform to the wavelet transform in the fusion of digital image and MR and CT images from entropy, difference entropy, quality measure, standard deviation, PSNR.
\end{abstract}

Keywords- Fusion, Wavelet transform, Curvelet Transform

\section{Introduction}

Image fusion has become a common term used within medical diagnostics and treatment. The term is used when multiple patient images are registered and overlaid or merged to provide additional information. Fused images may be created from multiple images from the same imaging modality, ${ }^{[1]}$ or by combining information from multiple modalities,[2] such as magnetic resonance image (MRI), computed tomography (CT).CT images are used more often to ascertain differences in tissue density while MRI images are typically used to diagnose brain tumors.

Multisensor data fusion has become a discipline which demands more general formal solutions to a number of application cases. Several situations in image processing require both high spatial and high spectral information in a single image. This is important in remote sensing. However, the instruments are not capable of providing such information either by design or because of observational constraints. One possible solution for this is data fusion.Image fusion is the process of merging two images of the same scene to form a single image with as much information as possible. Image fusion is important in many different image processing fields such as satellite imaging, remote sensing and medical imaging [2].Image fusion methods can be broadly classified into two groups - spatial domain fusion and transform domain fusion. The disadvantage of spatial domain approaches is that they produce spatial distortion in the fused image. Spectral distortion becomes a negative factor while we go for further processing, such as classification problem. Spatial distortion can be very well handled by frequency domain approaches on image fusion. The multiresolution analysis has become a very useful tool for analyzing remote sensing images. The discrete wavelet transform and curvelet transform based image fusion has become a very useful tool for medical and remote sensing images. These methods show a better performance in spatial and spectral quality of the fused image compared to other spatial methods of fusion. The images used in image fusion should already be registered.

Wavelet Transform has good time frequency characteristics. It was applied successfully in image processing field [3]. Nevertheless, itsexcellent characteristic in one-dimension can't be extended to two dimensions or multi-dimension simply. Separable wavelet which was spanning by onedimensional wavelet has limited directivity [4]. 
Aiming at these limitation, E. J. Candes and D. L. Donoho put forward Curvelet Transform theory in 2000 [5]. Curvelet Transform consisted of special filtering process and multi-scale Ridgelet Transform. It could fit image properties well. However, Curvelet Transform had complicated digital realization, includes sub-band division, smoothing block, normalization, Ridgelet analysis and so on. Curvelets pyramid decomposition brought immense data redundancy [6]. Then E. J. Candes put forward Fast Curvelet Transform(FCT) that was the Second Generation Curvelet Transform which was more simple and easily understanding in 2005[7]. Its fast algorithm was easily understood. Li Huihui's researched multi-focus image fusion based on the Second Generation Curvelet Transform [8]. This paper introduces the Second Generation Curvelet Transform and uses it to fuse images, different kinds of fusion methods are compared at last. The experiments show that the method could extract useful information from source images to fused images so that clear images are obtained.

\section{Image Fusion Based On Wavelet Transform}

The most common form of transform type image fusion algorithms is the wavelet fusion algorithm due to its simplicity and its ability to preserve the time and frequency details of the images to be fused.

Some generic requirements can be imposed on the fusion result. a) the fused image should preserve as closelyas possible all relevant information contained in the input images. b)The fusion process should not introduce any artefacts or in consistencies which can distract or mislead the human observer or any subsequent image processing steps. c) in the fused image irrelevant features and noise should be suppressed to a maximum extent. When fusion is done at pixel level the input images are combined withoutany pre-processing.

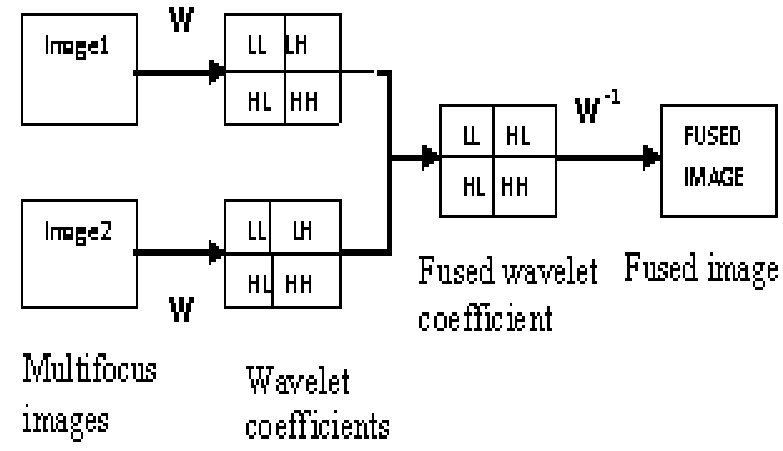

Fig.1 Block diagram of Discrete Wavelet transform

A schematic diagram of the wavelet fusion algorithm of two registered images $I_{1}\left(X_{1}, X_{2}\right)$ and $I_{2}\left(X_{1}, X_{2}\right)$ is depicted in fig.1. It can be represented by the following equation,

$\mathrm{I}\left(\mathrm{X}_{1}, \mathrm{X}_{2}\right)=\mathrm{W}^{-1}\left\{\Psi\left[\mathrm{W}\left(\mathrm{I}_{1}\left(\mathrm{X}_{1}, \mathrm{X}_{2}\right)\right), \mathrm{W}\left(\mathrm{I}_{2}\left(\mathrm{X}_{1}, \mathrm{X}_{2}\right)\right)\right]\right\}$

Where $\mathrm{W}, \mathrm{W}^{-1}$ and $\psi$ are the wavelet transformoperator, the inverse wavelet transform operator andthe fusion rule, respectively. There are several waveletfusion rules that can be used for the selection ofwavelet coefficients from the wavelet transforms of theimages to be fused. The most frequently used rule isthe maximum frequency rule which selects thecoefficients that have the maximum absolute values. The wavelet transform concentrates on representingthe image in multi-scale and it is appropriate torepresent linear edges. For curved edges, the accuracyof edge localization in the wavelet transform is low.So, there is a need for an alternative approach whichhas a high accuracy of curve localization such as thecurvelet transform.

\section{Types Of Wavelet Transforms}

A] Orthogonal Wavelet Transform

The dilations and translation of the scaling function $\phi \mathrm{j}, \mathrm{k}(\mathrm{x})$ constitute a basis for $\mathrm{Vj}$, andSimilarly $\Psi \mathrm{j}$, $\mathrm{k}(\mathrm{x})$ for $\mathrm{Wj}$, if the $\phi \mathrm{j}, \mathrm{k}(\mathrm{x})$ and $\Psi \mathrm{j}, \mathrm{k}(\mathrm{x})$ are orthonormal, they include the following property [1].

$$
\mathrm{Vj} \perp \mathrm{Wj}
$$

These results in a representation of a single image, containing multiscale detail information from all component images involved. This representation leads to multiple applications ranging from multispectral image fusion to color and multi-valued image enhancement, denoising and segmentation [9].

B] Bi-orthogonal Wavelet Transform

For biorthogonal transform, perfect reconstruction is available. Orthogonal wavelets give orthogonal matrices andunitary transforms; biorthogonal wavelets give invertiblematrices and perfect reconstruction. For biorthogonal wavelet filter, the Low-pass and high-pass filters do notthe same length. The low pass and high pass filters do nothave the same length. The low-pass filter is alwaysSymmetrical, while high pass filter could 
be eithersymmetric or anti-symmetric.The method allows unusual flexibility in choosinga filter for any task involving the multiresolution analysis and synthesis.Using our method, one can choose any low-pass filter forthe multiresolution filtering [1].

C]A'trous (Non-orthogonal) Wavelet Transform

A'trous is a kind of non - orthogonal wavelet that is different from orthogonal and biorthogonal. It is astationary or redundant transform, i.e. decimation is notimplemented during the process of wavelet transform, whilethe orthogonal or biorthogonal wavelet can be carried out using either decimation or undecimation mode [1].The enhancement of the spatial information often leadsto the distortion of the information in the spectral domain. Inthis paper, a spectral preserve fusion method is developed by introducing àtrous wavelet transform [10].

\section{Wavelet Transform Algorithm Steps}

The process can be divided into four steps.

a) Histogram match

Apply the histogram match process between panchromatic image and differentbands of the multispectral imagerespectively, and obtain three newpanchromatic images PANR, PANG,PANB

b) Wavelet decomposition

Use the wavelet transform to decomposenew panchromatic images and differentbands of multispectral image twice,respectively.

c) Details information combination

Add the detail images of the decomposed panchromatic images at different levels tothe corresponding details of differentbands in the multispectral image andobtain the new details component in thedifferent bands of the multispectral imageand obtain the new details component inthe different bands of the multispectralimage.

d) Inverse wavelet transform

Perform the wavelet transform on thebands of multispectral images,respectively and obtain the fused image.

\section{Image Fusion Based On Curvelet Transform}

The curvelet transform is a multiscale directional transform thatallows an almost optimal nonadaptive sparse representationof objects with edges. It hasgenerated increasing interestin the community of applied mathematics and signal processing over the years. Most natural images/signals exhibit line-like edges, i.e., discontinuities across curves (so-called line or curve singularities). Although applications of wavelets have become increasingly popular in scientific and engineering fields, traditional wavelets perform well only at representing point singularities since they ignore the geometric properties of structures and do not exploit the regularity of edges.

The curvelet transform has evolved as atool for the representation of curved shapes ingraphical applications. Then, it was extended to thefields of edge detection and image denoising. Recently, curvelet transform used in image fusion. Thealgorithm of the curvelet transform of an image P can be summarized in the following steps:

A) The image $\mathrm{P}$ is split up into three subbands $\Delta 1, \Delta 2$ and $\mathrm{P} 3$ using the additive wavelet transform.

B) Tiling is performed on the subbands $\Delta 1$ and $\Delta 2$.

C) The discrete Ridgelet transform is performed oneach tile of the subbands $\Delta 1$ and $\Delta 2$.

A schematic diagram of the curvelet transform isshown in Fig.2

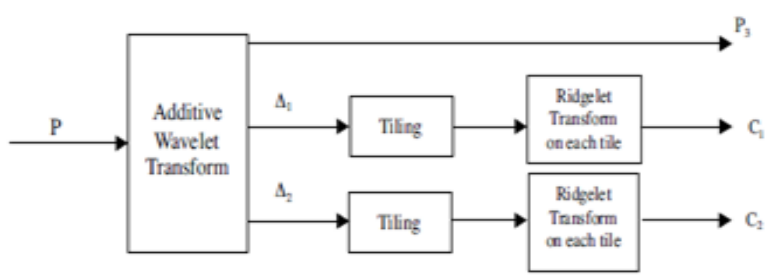

Figure 2. Discrete curvelet transform of an image $P$.

1. Subband Filtering:

The purpose of this step is to decompose the image into additive components; each of which is a subband of that image. This step isolates the different frequency components of the image into different planes without down sampling as in the traditional wavelet transform. Given an image $\mathrm{P}$, it is possible to construct the sequence of approximations: $F_{1}(P)=P_{1}, F_{2}(P)=P_{2}, \ldots \ldots \ldots F_{N}(P)=P_{N}$. Where $n$ is an integer which is preferred to be equal to 3 . To construct this sequence, successive convolutions with a certain low pass kernel are performed. The 
functions f1, f2, f3, and fn mean convolutions with this kernel. The wavelet planes are computed as the differences between two consecutive approximations $P_{1-1}$ and $P_{1}$ i.e. $\Delta 1=P_{1-1}-P_{1}$

Thus, the curvelet reconstruction formula is given by:

$\mathrm{n}-1$

$\mathrm{P}=\sum \Delta 1+\mathrm{P}_{1}$

$1=1$

2. Tiling:

Tiling is the process by which the image is divided into overlapping tiles. These tiles are small in dimensions to transform curved lines into small straight lines in the subbands $\Delta 1$ and $\Delta 2$. The tiling improves the ability of the curvelet transform to handle curved edges.

3. Ridgelet Transform:

The ridgelet transform belongs to the family of discrete transforms employing basis functions. Tofacilitate its mathematical representation, it can beviewed as a wavelet analysis in the Radon domain.The Radon transform itself is a tool of shape detection.So, the ridgelet transform is primarily a tool of ridgedetection or shape detection of the objects in an image.The ridgelet basis function is given by,

$\Psi_{\mathrm{a}, \mathrm{b}, \theta}\left(\mathrm{x}_{1}, \mathrm{x}_{2}\right)=\mathrm{a}^{-1 / 2} \Psi\left[\left(\mathrm{x}_{1} \cos \theta+\mathrm{x}_{2} \sin \theta-\mathrm{b}\right) / \mathrm{a}\right]$

for each $a>0$, each $\left[\begin{array}{lll}b & \varepsilon\end{array}\right]$ and each $\left(\begin{array}{lll}b & \varepsilon\end{array}[0,2 \pi]\right)$ this function constant along with lines $\mathrm{X}_{1} \cos \theta+\mathrm{X}_{2} \sin \theta=$ constant. Thus the ridgelet coefficients of an image $\mathrm{f}\left(\mathrm{x}_{1}, \mathrm{x}_{2}\right)$ are represented by:

$\infty \infty$

$\mathrm{R}_{\mathrm{f}}(\mathrm{a}, \mathrm{b}, \theta)=\iint \Psi_{\mathrm{a}, \mathrm{b}, \theta}\left(\mathrm{x}_{1}, \mathrm{x}_{2}\right) \mathrm{f}\left(\mathrm{x}_{1}, \mathrm{x}_{2}\right) \mathrm{dx}_{1} \mathrm{dx}_{2}$

$-\infty-\infty$

This transform is invertible and the reconstruction formula is given by:

$2 \pi \infty \infty$

$\mathrm{f}\left(\mathrm{x}_{1}, \mathrm{x}_{2}\right)=\iint \mathrm{R}_{\mathrm{f}}(\mathrm{a}, \mathrm{b}, \theta) \Psi_{\mathrm{a}, \mathrm{b}, \theta}\left(\mathrm{x}_{1}, \mathrm{x}_{2}\right) \mathrm{dadbd} \theta$

$0-\infty 04 \pi \mathrm{a}$ is given by:

The radon transform for an object $F$ is the collection of line integrals indexed by $(\theta, t) \varepsilon[0,2 \pi] \times R$ and

$\infty \infty$

$R_{f}(\theta, t)=\iint f\left(x_{1}, x_{2}\right) \delta\left(x_{1} \cos \theta+x_{2} \sin \theta-t\right) d x 1 d x 2$

$-\infty-\infty$

Thus for ridgelet transform can be represented in terms of the randon transform as follows:

$\infty$

$\mathrm{R}_{\mathrm{f}}(\mathrm{a}, \mathrm{b}, \theta)=\int \mathrm{R}_{\mathrm{f}}(\theta, \mathrm{t}) \mathrm{a}^{-1 / 2}[(\mathrm{t}-\mathrm{b}) / \mathrm{a}] \mathrm{dt}$

$-\infty$

Hence, the ridgelet transform is the application of the1-D wavelet transform to the slices of the Radontransform where the angular variable $\theta$ is constant and it is varying.To make the ridgelet transform discrete,both the Radon transform and the wavelet transformhave to be discrete.It is known that different imaging modalities areemployed to depict different anatomical morphologies.CT images are mainly employed to visualize densestructures such as bones. So, they give the generalshapes of objects and few details. On the other hand,MR images are used to depict the morphology of softtissues. So, they are rich in details. Since these twomodalities are of a complementary nature, ourobjective is to merge both images to obtain as muchinformation as possible.

\section{Wavelet And Curvelet Based Image Fusion Algorithm}

1. First, we need pre-processing, and then cut the same scale from awaiting fused images according to selected region. Subsequently, we divide images into sub-images which are different scales by WaveletTransform. Afterwards, local Curvelet Transform of every sub-image should be taken. Its sub-blocks aredifferent from each others on account of scales' change.

2. Resample and registration of original images, we can correct original images and distortion so that bothof them have similar probability distribution. Then Wavelet coefficient of similar component will stay in the same magnitude.

3. Using Wavelet Transform to decompose original images into proper levels. One low-frequency approximate component and three high-frequency detail components will be acquired in each level.

4. Curvelet Transform of individual acquired low frequency approximate component and high frequencydetail components from both of images, neighborhood interpolation method is used and the details of graycan't be changed.

5. According to definite standard to fuse images, local area variance is chose to measure definition for low frequency component. First, divide low-frequency coefficients $C$ jo(k1,k2) into individual four square 
subblocks which are N1X M2 ( $3 \times 3$ or $5 \times 5$ ), then calculate Standard deviation(STD) of the current sub-block and other statistical parameters.

\section{Results}

In medicine, CT and MRI image both are tomography scanning images. They have different features. Fig. 3 shows CT image, in which image brightness related to tissue density, brightness of bones is higher, and some soft tissue can't be seen images. Fig. 4 shows MRI image, here image brightness related to an amount of hydrogen atom in tissue, thus brightness of soft tissue is higher, and bones can't be seen. There is complementary information in these images.

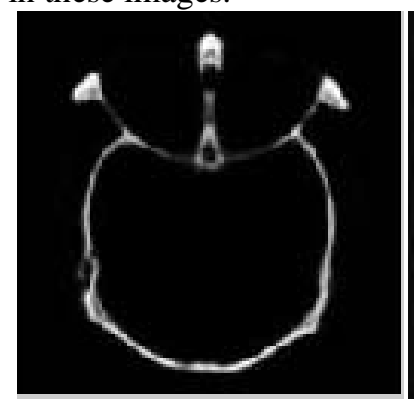

Fig. 3 CT Image of brain

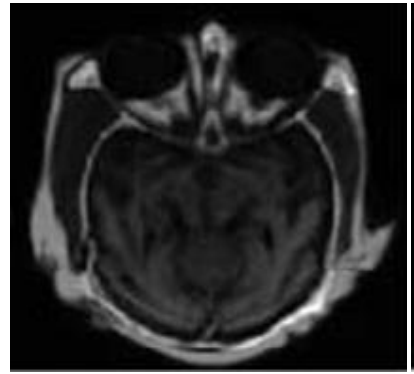

Fig.5a) Orthogonal Fused Image

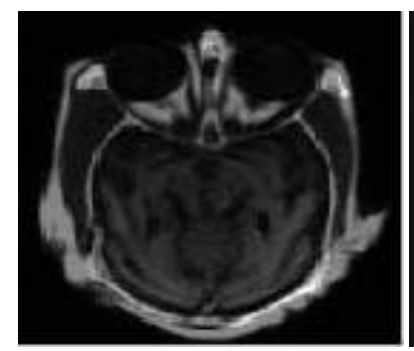

Fig. 5c)A’trous fused Fig.6 Fused Image of image

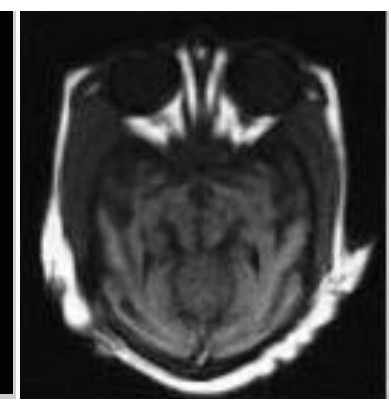

Fig. 4 MRI Image of brain

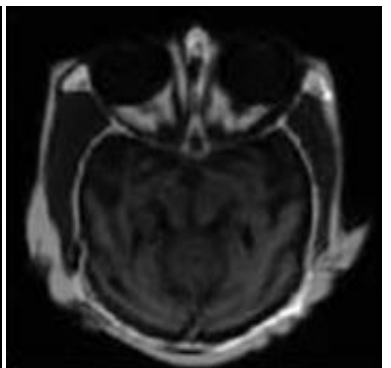

Fig.5b) Biorthogonal

Fused Image

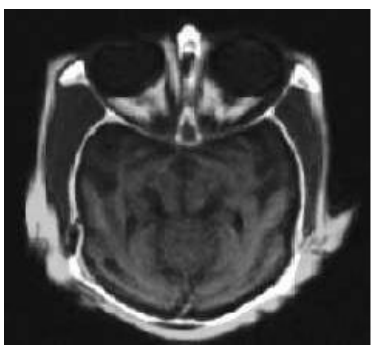

Wavelet Transform

Fig. 3 and fig. 4 represents the MRI and CT images of brain of same person respectively.In the MRI image theinner contour missing but it provides better information onsoft tissue. In the CT image it provides the best informationon denser tissue with less distortion, but it misses the softtissue information. The fig. 5a image is the result of orthogonal waveletfusion technique which is by combining of MRI and CT images .The orthogonal wavelet fused image haveinformation of both images but have more aliasing effect. The fig. 5b image is the result of Biorthogonal wavelet fusion technique. When compare Biorthogonal wavelet withorthogonal wavelet it shows soft tissues information whichare not shown in above figure i.e at the left and right side ofthe inner part. The fig. 5c image is the result of 'A trous' wavelet(non-orthogonal wavelet) based fusion. The fusion results of non-orthogonal wavelet have information on soft tissues anddenser tissues. 


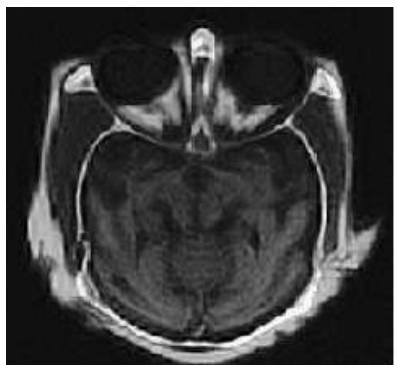

Fig.7 Fused Image of

Curvelet Transform

Since the curvelet transform is well-adapted to represent panchromatic image containing edges as shown in fig.7 and the wavelet transform preservesspectral information of original multispectral images as shown in fig.6, the fusedimage has high spatial and spectral resolution simultaneously. In addition to the visual analysis, we extended our investigationto a quantitative analysis. The experimental result was analyzedbased on the combination entropy, standard deviation, quality measure as shown in table 1.

\section{Quantitative Analysis}

In order to compare the wavelet and curvelet based approaches; apart from visual appearancequantitative analysis is done over the fused images. For the visual evaluation, the followingcriterion is considered: natural appearance, brilliance contrast, presence of complementaryfeatures, enhancement of common features etc. The quantitative criterion [11] includes three parameters namely Entropy, DifferenceEntropy and Standard deviation. Each has its importance in evaluating the image quality.

1. Entropy: The entropy of an image is a measure of information content . The estimate assumes a statistically independent source characterized by the relative frequency of occurrence of the elements in X, which is its histogram. For a better fused image, the entropy should have a larger value.

2. Difference Entropy: It is calculated from taking the entropy of the image obtained from subtracting a source image from the fused image and the input source image.

Example: Fused image -CT Image=MRI ImageEntropy [obtained MRI Image - Input MRI] gives Difference Entropy. The difference entropy between two images reflects the difference between the average amounts of information they contained. Minimum difference is expected for a better fusion.

3. Standard deviation: The standard deviation (SD), which is the square root of variance, reflects the spread in the data. Thus, a high contrast image will have a larger variance, and a low contrast image will have a low variance.

Table 1: Statistical parameters of Wavelet and Curvelet transform

\begin{tabular}{|c|c|c|}
\hline $\begin{array}{c}\text { FUSION } \\
\text { METHODS }\end{array}$ & $\begin{array}{c}\text { WAVELET } \\
\text { TRANSFOM }\end{array}$ & $\begin{array}{c}\text { CURVELET } \\
\text { TRANSFORM }\end{array}$ \\
\hline Entropy & 5.05 & 5.823 \\
\hline $\begin{array}{c}\text { Difference } \\
\text { entropy }\end{array}$ & 5.40 & 5.361 \\
\hline $\begin{array}{c}\text { Standard } \\
\text { deviation }\end{array}$ & 62.03 & 69.29 \\
\hline $\begin{array}{c}\text { Quality measure } \\
\text { Q }\end{array}$ & 0.891 & 0.90 \\
\hline RMSE & 2.392 & 1.530 \\
\hline
\end{tabular}

Quantitative analysis of the fused images indicates better results for curvelet transform based fusion with greater entropy, larger standard deviation and lower difference entropy than their wavelet equivalents. And among the curvelets, addition gives a better result.Moreover, compared withthe fused results obtained by the wavelet and the curvelet, the curveletbasedfusion result has a better visual effect, such as contrastenhancement. 


\section{Conclusion}

A comparison study has been made between the traditional wavelet fusion algorithm and the proposed curvelet fusion algorithm. The experimental study shows that the application of the curvelet transform in the fusion of MR and CT images is superior to the application of the traditional wavelet transform.In many important imaging applications, images exhibit edges anddiscontinuities across curves. In biologicalimagery, this occurs whenever two organs ortissue structures meet. Especially in image fusionthe edge preservation is important in obtainingthe complementary details of the input images. As edge representation in Curvelet is better,Curvelet based image fusion is best suited formedical images.

\section{References}

[1] A. Soma Sekhar, Dr.M.N.Giri Prasad, A Novel Approach of Image Fusion on MR and CT Images Using Wavelet Transforms, 2011 IEEE

[2] Smt.G. Mamatha(Phd), L.Gayatri, An Image Fusion Using Wavelet And Curvelet TransformGlobal Journal of Advanced Engineering Technologies, Vol1, Issue-2, 2012 , ISSN: 2277-6370

[3] Abhijit Somnathe, Ujwal Harode, A Novel Approach of Image Fusion based on Wavelet Transform and Curvelet Transform,International Conference \& Workshop on Recent Trendsin Technology, (TCET) 2012

[4] Rafael c. Gonzalez,richard E.woods Digital image processing, Addison-wesley.an imprint of pearson education, 1st edition

[5] E. J. Candes, D. L. Donoho. Curvelets: A surprisingly effective nonadaptive representation for objects with edges[J]. In:C. Rabut A. Cohen, L. L. Schumaker. Curves and Surfaces. Nashville, TN: Vanderbilt University Press ,2000. 105-120.

[6] E. J. Candes, D. L. Donoho. New tight frames of curvelets and optimal representations of objects with singularities[J]. Commun. On Pure and Appl. Math.2004, 57(2):219-266.

[7] E. J. Candes , L. Demanet D. L. Donoho et al.. Fast Discrete Curvelet Transforms[R].Applied and Computational Mathematics. California Institute of Technology , 2005.1.

[8] LiHui-hu,i GuoLe,i LiuHang. Research on image fusion based on the second generation curvelet transform [J]. Acta Optica Sinica, 2006,26(5): $657 \sim 662$

[9] Paul Scheunders, Member, IEEE. An Orthogonal Wavelet Representation ofMultivalued Images. IEEE Transactions on Image Processing, Vol. 12, no. 6, June 2003

[10] Shaohui Chen, Renhua Zhang, Hongbo Su, Senior Member, IEEE, Jing Tian, and Jun Xia,SAR and Multispectral Image Fusion Using Generalized IHS Transform Based on à Trous Wavelet and EMD Decompositions. IEEE SENSORS JOURNAL, VOL. 10, NO. 3, MARCH 2010

[11] Y.Kiran Kumar Technical Specialist- Philips HealthCare, Comparison Of Fusion Techniques Applied To Preclinical Images: Fast Discrete Curvelet Transform Using Wrapping Technique \& Wavelet Transform.Journal of Theoretical and Applied Information Technology 